\title{
Erythema Multiforme Major in Angioimmunoblastic T-cell Lymphoma
}

Shoko HATTORI, Fumi MIYAGAWA*, Keiko FUKUDA, Kohei OGAWA and Hideo ASADA

Department of Dermatology, Nara Medical University School of Medicine, 840 Shijo, Kashihara, Nara 634-8522, Japan. E-mail: fumim@ naramed-u.ac.jp

Accepted Dec 8, 2021; Epub ahead of print Dec 8, 2021

Angioimmunoblastic T-cell lymphoma (AITL) is a distinct peripheral T-cell lymphoma of the follicular Thelper cell lineage (1). Skin rashes are reported to occur in approximately half of cases, but non-specific rashes, such as maculopapular rashes without histopathological atypical cell infiltration, are common (2-4). The nonspecific clinical and histological findings of AITL make its diagnosis very challenging (4).

We report here a case of AITL, involving widespread target-shaped erythematous plaques with central blisters, accompanied by mild mucosal lesions and fever. These symptoms led us to suspect erythema multiforme major (EMM), but the patient was subsequently diagnosed with AITL with clonal T-cell receptor (TCR) gene rearrangement affecting both the skin and lymph nodes (LNs). To our knowledge, this is the first documented case of EMM occurring as a specific rash in AITL.

\section{CASE REPORT}

An 80-year-old Japanese woman developed erythema on her body, which resolved after treatment with topical steroid. Three months later, she presented with a fever of $38.4^{\circ} \mathrm{C}$, general malaise, a cough, and anorexia, as well as generalized erythema with pruritus. A physical examination revealed numerous target-shaped erythematous and violaceous plaques on her face, trunk, and extremities (Fig. 1a-c). The violaceous plaques on her legs and feet had central bullae (Fig. 1d). Erosions were noted in the oral mucosa and genital region, but they were not haemorrhagic. The Nikolsky sign was negative. The cervical and inguinal LNs were palpable.

She had had a gastric ulcer for a few years, which had been treated with rabeprazole. She had not started taking any new drugs recently. Blood tests showed the following abnormalities: while blood cells $221 \times 10^{2} /$ $\mu 1$ (normal range 33-86), red blood cells $313 \times 10^{4} / \mu 1$ (normal range 386-492), haemoglobin $10.2 \mathrm{~g} / \mathrm{dl}(11.6-14.8)$, haematocrit $31.1 \%$ (35.1-44.4), lymphocytes $5.0 \%$ (16.5-49.5) C-reactive protein $7.54 \mathrm{mg} / \mathrm{dl}$ $(<0.14)$, albumin $2.9 \mathrm{~g} / \mathrm{dl}(4.1-5.1)$, globulin $3.8 \mathrm{~g} / \mathrm{dl}(2.2-3.4)$, lactate dehydrogenase 436
$\mathrm{U} / 1$ (124-222), blood urea nitrogen $41 \mathrm{mg} / \mathrm{dl}$ (8-20), and creatinine $2.01 \mathrm{mg} / \mathrm{dl}(0.46-0.79)$.

Histopathological examination of the erythema on the left forearm revealed scattered necrotic keratinocytes in the epidermis and dense infiltrates of lymphocytes, histiocytes, neutrophils, and eosinophils in the dermis. Extravasated erythrocytes and nuclear debris were also seen in the dermis (Figs 1e and f). No atypical lymphocytes were observed. Immunohistochemistry revealed that the majority of the infiltrates were composed of $\mathrm{CD}^{+} \mathrm{CD}^{+}$
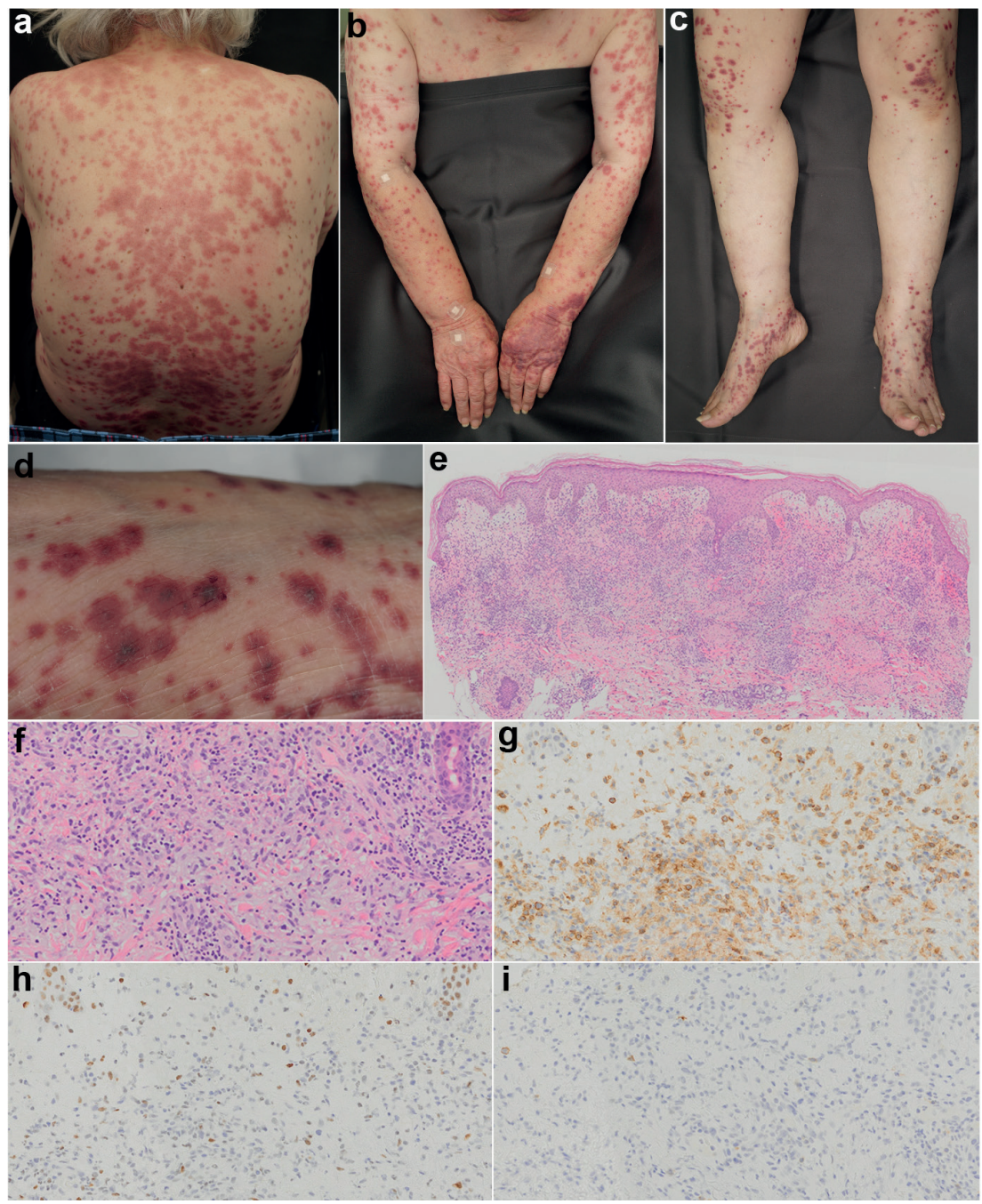

Fig. 1. Widespread erythematous or purpuric macules on: (a) the back, (b) upper extremities, and (c) lower extremities. (d) Close-up view of the patient's right leg. Purpuric target-shaped lesions with central blisters were visible. (e) A biopsy specimen from the left forearm revealed scattered necrotic keratinocytes in the epidermis and dense lymphohistiocytic infiltrates in the dermis (haematoxylin \& eosin staining; $\times 40$ ). (f) Magnified view of $(e)$. There were dense infiltrates of lymphocytes, histiocytes, neutrophils, and eosinophils in the dermis (haematoxylin \& eosin staining; $\times 200)$. $(\mathrm{g}-\mathrm{i})$ Immunohistochemistry demonstrated that the majority of the infiltrates were positive for CD4 $(g)(\times 200)$. Scattered small lymphocytes were positive for $(h)$ Bcl-6 and (i) PD-1 $(\times 200)$. 
cells (Fig. 1g), and scattered small lymphocytes were positive for CD10, Bcl-6 (Fig. 1h), and programmed cell death-1 (PD-1) (Fig. 1i). Epstein-Barr virus (EBV)-encoded small non-polyadenylated RNA (EBER) was not detected.

A computed tomography (CT) scan demonstrated hepatosplenomegaly and numerous enlarged LNs in the neck, axilla, mediastinum, abdominal cavity, and inguinal regions.

A diagnosis of EMM was made, and treatment with intravenous methylprednisolone ( $500 \mathrm{mg} /$ day for 3 days) was started. The skin lesions disappeared rapidly, and then the treatment was switched to $60 \mathrm{mg}$ /day oral prednisolone. At the same time, as the patient demonstrated significant lymphadenopathy, a biopsy from the inguinal LNs was performed. Effacement of the LN architecture due to the proliferation of atypical lymphoid cells with pale cytoplasm was noted. Eosinophilic infiltrates and an increased number of arborizing vessels were also observed. Immunohistochemistry demonstrated that most of the atypical lymphoid cells were positive for CD3, CD4, CD10, Bcl-6, and PD-1. The scattered small lymphocytes were positive for EBER. PCR-based analysis of formalin-fixed paraffin-embedded LN biopsy tissue demonstrated clonal rearrangement of both the TCR $\beta$-chain and immunoglobulin heavy-chain genes. A diagnosis of AITL was eventually made, and THP-COP therapy was started immediately, which produced a favourable response.

We subsequently re-evaluated the skin biopsy using PCR analysis of formalin-fixed paraffin-embedded tissue and found clonal rearrangement of the TCR $\beta$-chain gene.

\section{DISCUSSION}

AITL is clinically characterized by generalized lymphadenopathy; hepatosplenomegaly; systemic symptoms, such as fevers, weight loss, and night sweats; and polyclonal hypergammaglobulinaemia $(1,4)$. Typically, histological examinations of the LNs reveal pleomorphic infiltration, including by neoplastic $\mathrm{T}$ cells and non-neoplastic lymphocytes, plasma cells, eosinophils, and epithelioid histiocytes, accompanied by the marked proliferation of high endothelial venules and follicular dendritic cells $(1,4)$. Secondary EBV-positive B-cell proliferation and expanding B-cell clones are also often seen (1).

Approximately $50 \%$ of patients with AITL also have cutaneous manifestations. These involve maculopapular rashes in most cases, but erythematous, erythrodermic, plaque, purpuric, urticarial, nodular, petechial, or papulovesicular lesions can also occur (2-4). Cutaneous involvement is often related to clonal T-cell proliferation, even when the observed clinical and histological features are non-specific (2), and a review of the cutaneous manifestations of AITL indicated that TCR $\gamma$ gene rearrangement was detected in the skin of 20 of the 23 cases examined $(87 \%)(4)$. There is only 1 previous report of target-shaped erythematous lesions mimicking $\operatorname{EMM~(5),~as~was~seen~in~}$ the current case. Zhang et al. (5) reported a case of AITL in which infectious mononucleosis and haemophagocytic lymphohistiocytosis developed at the same time as an EMM-like skin rash. However, they did not examine the skin rash for clonal TCR gene rearrangement (5). In the current case, although atypical lymphocytic cell infiltrates were not obvious in the skin biopsy sample, and only a few scattered lymphocytes, which were positive for CD10, Bcl-6, and PD-1, were seen, the presence of clonal TCR gene rearrangement suggests that the EMM-like skin rash was directly caused by AITL.

Target-shaped skin lesions associated with StevensJohnson syndrome (SJS)/toxic epidermal necrolysis (TEN) have also been reported in 1 previous case of AITL (6). In the latter case, a 67-year-old male initially presented with lymphadenopathy, fever, and erythrodermic spongiotic dermatitis and subsequently developed SJS/TEN. Although the skin lesions subsided after treatment with oral prednisolone and intravenous immunoglobulins, the lymphadenopathy persisted, and a LN biopsy led to a diagnosis of AITL 1 month after the development of TEN (6). Whether the SJS/TEN in this case was directly related to the AITL was not clear. As it has now been established that EMM is a distinct disease entity that differs from SJS/TEN (7) and that it is not clear whether the EMM-like skin rash seen in the AITL patient reported by Zhang et al. was specifically caused by AITL, the current case appears to be the first documented case in which EMM occurred as a specific manifestation of AITL. Since the cutaneous manifestations of AITL vary significantly, AITL should be strongly suspected in cases in which EMM is associated with a fever and lymphadenopathy, even if no evidence of lymphoma is detected during a skin biopsy, as we misdiagnosed the current case as EMM after the skin biopsy and only correctly diagnosed AITL after a LN biopsy and CT scan.

The authors have no conflicts of interest to declare.

\section{REFERENCES}

1. Swerdlow SH, Campo E, Harris NL, Jaffe ES, Pileri SA, Stein $\mathrm{H}$, et al. Angioimmunoblastic T-cell lymphoma. In: WHO classification of tumours of haematopoietic and lymphoid tissues. IARC; 2017; p. 408-410.

2. Martel P, Laroche L, Courville P, Larroche C, Wechsler J, Lenormand $B$, et al. Cutaneous involvement in patients with angioimmunoblastic lymphadenopathy with dysproteinemia: a clinical, immunohistological, and molecular analysis. Arch Dermatol 2000; 136: 881-886.

3. Botros N, Cerroni L, Shawwa A, Green PJ, Greer W, Pasternak $\mathrm{S}$, et al. Cutaneous manifestations of angioimmunoblastic T-cell lymphoma: clinical and pathological characteristics. Am J Dermatopathol 2015; 37: 274-283.

4. Balaraman B, Conley JA, Sheinbein DM. Evaluation of cutaneous angioimmunoblastic T-cell Iymphoma. J Am Acad Dermatol 2011; 65: 855-862.

5. Zhang L, Tong C, Tan Y, Peng S, He Y, Wang T. Hemophagocytic lymphohistiocytosis presenting with annular erythema multiforme-like eruptions in a patient with angioimmunoblastic T cell lymphoma: a case report. Exp Ther Med 2018; 16: 2060-2065.

6. Jones B, Vun Y, Sabah M, Egan CA. Toxic epidermal necrolysis secondary to angioimmunoblastic T-cell lymphoma. Australas J Dermatol 2005; 46: 187-191.

7. Auquier-Dunant A, Mockenhaupt M, Naldi L, Correia O, Schroder W, Roujeau JC, et al. Correlations between clinical patterns and causes of erythema multiforme majus, StevensJohnson syndrome, and toxic epidermal necrolysis: results of an international prospective study. Arch Dermatol 2002; 138: 1019-1024. 